\title{
Identifying Common Risk factors for Cancer, Diabetes and Hypertension: Nursing Perspective
}

\author{
Lt Col ( Retd) M Jayalakshmi, \\ Principal, Shri Vinoba Bhave College of Nursing SVBCH CAMPUS, Silvassa, DNH-UT. Pin -396230.
}

\begin{abstract}
Chronic diseases are by far the leading cause of mortality in the world, representing $63 \%$ of all deaths. Out of the 36 million people who died from chronic disease in 2008, nine million were under 60 and ninety per cent of these premature deaths occurred in low- and middle-income countries" [WHO] 2011. In India, their burden will continue to increase during the next 25 years as a consequence of the rapidly ageing population in India. Most chronic diseases are common and often occur as co morbidities. Risk factors for chronic diseases are highly prevalent among the Indian population. The study aimed to identify the common risk factors that are prevalent among the cervical cancer Patients and the opportunities for nurses in primary and secondary prevention and managing chronic diseases. A total of 100 records were reviewed who have completed the treatment for cervical cancer. The main focus was on the prevalence of common co morbidities, obesity, dietary factors and consumption of tobacco and alcohol. The study identified the common risk factors for the con communicable diseases ie cancer, diabetes, hypertension and cardiac conditions are overweight, type of food consumption, tobacco usage and alcohol. Nurses are in the ideal position in trying to preventing the non communicable diseases and maintain people to prevent major complications of the diseases.
\end{abstract}

Key Words: chronic diseases, common risk factors, nursing perspective

\subsection{Background of the study}

\section{Introduction}

Non communicable diseases (NCDs) are the leading causes of death globally, killing more people each year than all other causes combined. Nearly $80 \%$ of NCD deaths occur in low- and middle-income countries. Of the 57 million deaths that occurred globally in 2008, 36 million - almost two thirds - were due to NCDs, comprising mainly cardiovascular diseases, cancers, diabetes and chronic lung diseases. Chronic diseases are the leading cause of death and disability worldwide. They account for almost $60 \%$ of all deaths and $43 \%$ of the global burden of disease [1]. The number of adults with diabetes is expected to increase from 366 million in 2011 to 552 million by 2030 [2]. It is expected to markedly increase with chronic disease contributing $73 \%$ of all deaths and $60 \%$ of the global burden of disease by 2020 [1]. In India, chronic diseases rising from 3.78 million in 1990 (40.4\% of all deaths) to an expected 7.63 million in 2020 (66.7\% of all deaths) due to the rapidly ageing population. Most chronic diseases are common and often occur as co morbidities. Risk factors for chronic diseases are highly prevalent among the Indian population. About one fourth of global NCD-related deaths take place before the age of 60 .

Non Communicable Diseases (NCDs) are caused, to a large extent, by four behavioral risk factors that are pervasive aspects of economic transition, rapid urbanization and 21st-century lifestyles: tobacco use, unhealthy diet, insufficient physical activity and the harmful use of alcohol. The greatest effects of these risk factors fall increasingly on low- and middle-income countries, and on poorer people within all countries, mirroring the underlying socioeconomic determinants. Among these populations, a vicious cycle may ensue: poverty exposes people to behavioral risk factors for NCDs and, in turn, the resulting NCDs may become an important driver to the downward spiral that leads families towards poverty.

1.2. Aim : The study aimed to identify the common risk factors that are prevalent among the cervical cancer Patients and the opportunities for nurses in primary and secondary prevention and managing chronic diseases.

\section{Materials and Methods}

Records of patients who were admitted in the period from Jan to Mar 2013 were reviewed. A total of 100 records were reviewed who have completed the treatment for cervical cancer. The main focus was on the prevalence of common co morbidities and common risk factors like obesity, dietary factors and consumption of tobacco and alcohol .

\section{Results}

Majority of participants ( $80 \%$ ) belonged to stage 2 ca cervix and $7 \%$ of patients had family history of cancer. The age of the participants ranged from 29 to 75 yrs with a mean age of 51.35.All participants were 
married with the mean age at marriage of 17.55 . A maximum of $36 \%$ of subjects had 3 children, while 2 subjects had 9 children (TABLE 1).

Table 1. Mean values of Age, number of children , BMI ,Age at marriage of participants

\begin{tabular}{|l|l|l|l|l|}
\hline & min & max & mean & Std mean \\
\hline Age & 29 & 75 & 51.35 & 10.061 \\
\hline $\begin{array}{l}\text { Age at } \\
\text { marriage }\end{array}$ & 11 & 28 & 17.57 & 3.358 \\
\hline \begin{tabular}{l} 
Children \\
\hline BMI
\end{tabular} & 0 & 9 & 3.61 & 1.687 \\
\hline & 15.11 & 45.88 & 24.65 & 4.90 \\
\hline
\end{tabular}

Majority of the participants were from rural place and belonged to the lower income group..Fruit was consumed daily only by 6 participants while 43 patients were not consuming fruit at all.(Fig.1.). Common co morbidities (Fig.2.) found were type II DM (5\%), diabetes with hypertension (9\%), DM,HTN and IHD (1\%),Psychiatric disorder with hypothyroidism(2\%).

Fig 1 .Dietary habits of participants
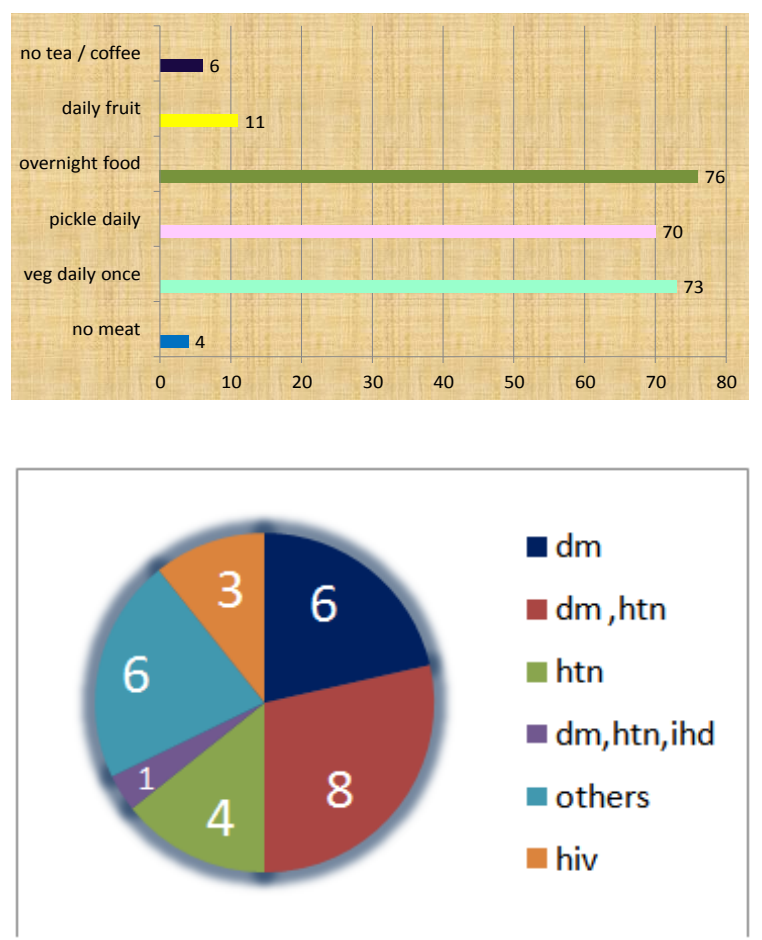

Fig.2. Presence of comorbidities among cervical Cancer patients 


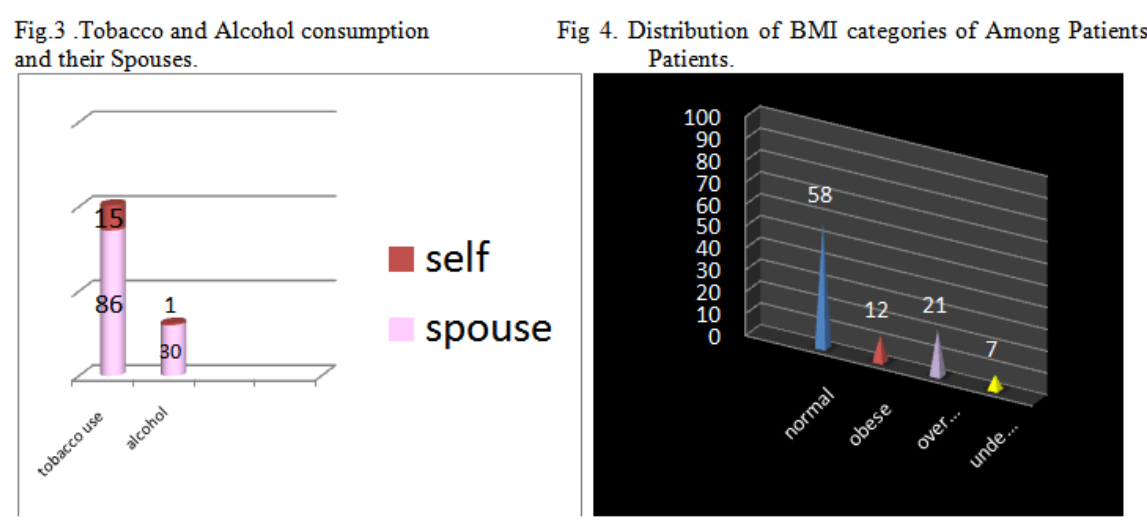

One patient was found alcohol dependent and had discontinued treatment. Fourteen patients were in the habit of using tobacco. (Fig.3.) Average BMI of participants was 24.46 while $26 \%$ of them were found overweight and $16 \%$ were obese. (Fig.4.)

\section{Discussion}

Most of the risk factors emerge during middle age as a result of an unhealthy lifestyle that has been followed for several decades. Many patients have several risk factors. In Nigeria it was found that patients may have as many as five chronic disease risk factors [ 3] The most prevalent chronic diseases - cardiovascular diseases, cancer, and type 2 diabetes - are linked by common and preventable biological risk factors (i.e., high blood pressure, dyslipidemia, and obesity) as well as major behavioral risk factors including unhealthy diet, physical inactivity, and tobacco use. [4] Patient centered care, which includes self-care or self-management, is a fundamental concern for nursing and essential to the prevention and management of chronic diseases [4].

Life style and environmental factors play an important role in the prevention of chronic disease particularly tobacco use, physical inactivity and poor diet .Christine Hancock has recommended that nursing curricula need to focus on the competencies to equip nurses to intervene early and support, encourage life style change and behavior modification. [5]The present has study identified the common risk factors for the con communicable diseases ie cancer, diabetes, hypertension and cardiac conditions are overweight, type of food consumption, tobacco usage and alcohol. The two biggest challenges in chronic disease are preventing the disease in the first place, and managing the condition effectively when it arises. Both of these are long-term challenges that are best addressed through an effective and ongoing relationship between the health-care provider and the patient, based on mutual respect, trust and appropriate interventions. The primary care setting is where this long-term relationship is most often established and where interventions occur.

\section{Conclusion}

Several groups of chronic diseases often occur together and have similar health-system interventions Common risk factors often present in diabetes, coronary heart disease, stroke, and chronic obstructive pulmonary disease and cancers especially breast, cervical, and lung cancer. Nurses are in the ideal position in trying to preventing the non communicable diseases and maintain people in a secondary prevention mode.

\section{Ackowledgement}

Chairman, Cancer Institute (WIA), Adyar Chennai

\section{References}

[1]. World Health Organization. Global Status Report on Non communicable Diseases 2010: Description of the Global Burden of NCDs, Their Risk Factors and Determinants. Geneva, Switzerland: WHO; 2011.

[2]. Unwin N, Whiting D, Guariguata L, Ghyoot G, Gan G. The IDF Diabetes Atlas. 5th edition. Brussels, Belgium: International Diabetes Federation; 2011.

[3]. Jamison DT, Feachem RG, Makgoba MW, et al., editors. Disease and mortality in sub-saharan Africa Washington (DC): World Bank; 2006. 2nd edition.

[4]. Lorig KR, Ritter PL, Laurent DD, Plant K. Internet-based chronic disease self-management: a randomized trial. Medical Care. 2006;44(11):964-971.

[5]. Nurses leading the fight against chronic disease .Report of a meeting held at the headquarters of the American Nurses Association on 24-25 June, 2010.

Ethical and SAC approvals received.

[6]. Interest of conflict: Nil.

[7]. Sources of finance of the Project : self. 